\title{
Vision screening, ocular disease assessment, and referral patterns of primary care providers within a Graduate Medical Education system
}

Reece Bergstrom ( $\nabla$ reece.bergstrom@gmail.com )

Ohio University https://orcid.org/0000-0002-0735-224X

Craig Czyz

Ohio University

Jeff Hutchison

Ohio University

\section{Research Article}

Keywords: GME, ophthalmology evaluation, American College of Physician (ACP), ACGME

Posted Date: March 13th, 2021

DOI: https://doi.org/10.21203/rs.3.rs-307246/v1

License: (c) This work is licensed under a Creative Commons Attribution 4.0 International License. Read Full License 


\section{Abstract}

Background Vision related screening procedures, ocular disease assessment, and ophthalmologic referral patterns of family (FM) and internal medicine (IM) residents and attendings, within a Graduate Medical Education (GME).

Objective The study evaluated FM \& IM residents' and attendings' ophthalmic evaluation practices, knowledge base, and referral patterns within a GME ambulatory care network.

Methods An anonymous survey was conducted in 2018 determine the incidence and methods of visual screening and what patient symptoms, medical histories, or complaints prompted referral to an ophthalmologist. The provider's knowledge base of ocular diseases and systemic treatments with potential ocular complications was also assessed. The referral patterns of the FM/IM providers for ophthalmologic care was evaluated for rationale and location.

Results The survey response rate was $45 \%$. A low percentage of providers (16\%) indicated ocular diseases was part of their past medical history evaluation. Nearly half the respondents $(40 \%)$ indicated they did not assess the visual status of their patients. When patients reported visual complaints or visual deficiency was noted on exam, all providers (100\%) indicated they refer patients for ophthalmology evaluation. There was a significant percentage of providers that indicated they did not know where to refer patients for ophthalmology evaluation (42\%).

Conclusions This analysis presents the opportunity to improve a GME primary care team's visual screening practices to improve care access and quality for patients requiring ophthalmologic services with a timely and appropriate referral. Similarly, further education recommendations provided to practitioners regarding specific indicators requiring ophthalmologic referral.

\section{Introduction}

Vision related screening procedures, ocular disease assessment, and ophthalmologic referral patterns of family (FM) and internal medicine (IM) residents and attendings, within a Graduate Medical Education (GME) ambulatory care network has previously not been detailed. Additionally, no study has assessed primary care providers' knowledge base related to common ocular diseases within a GME training system. The primary risk factors for many common ocular diseases are related to systemic conditions evaluated and managed by primary care physicians. Additional risk factors are often related to patient history, a primary component of an annual or new patient medicine clinic visit. Therefore, it is important for FM \& IM providers to not only include ocular concerns in their medical history and review of systems, but understand potentially associations of systemic conditions and medications to ocular disease.

The American Academy of Ophthalmology (AAO) recommends individuals over the age of 40 have a complete, dilated eye examination and those 65 or older are examined every 1 or 2 years ${ }^{1}$. However, individuals with risk factors for eye disease or those with visual symptoms, should have a baseline ophthalmologic evaluation regardless of age ${ }^{1}$. The AAO identifies the following specific risk factors for eye disease: diabetes (DM), hypertension (HTN), and family history of eye disease. ${ }^{1}$

The United States Preventive Services Task Force does not list visual screening in either it's A or B recommendations for adults. ${ }^{2}$ The American Academy of Family Physicians (AAFP) does not include visual screening as part of its adult "well examination" for either males or females. ${ }^{3,4}$ Similarly, the American College of Physician (ACP) does not provide any visual screening guidelines. ${ }^{5}$ While the utility of visual screening in healthy adults under the age of 65 can be questioned, those with systemic diseases that have significant impact on end organs certainly would benefit from screening. None of the previously referenced practice guidelines indicate the need for visual screening in patients with known systemic disease that have potential visual implications such has hypertension or diabetes.

Page $2 / 9$ 
The disparity in recommendations between various medical specialty colleges is not unique to vision related concerns. This disparity can also be present between specialty colleges and Accreditation Council for Graduate Medical Education (ACGME) residency training program requirements. For example, the AAFP's Recommended Curriculum Guidelines for Family Medicine Residents (RCGFMR) lists various competencies including the ability to perform basic vision screening and examination, indications for screening examinations in the general population and in patients who have systemic disease, and an understanding of the ophthalmology consultants role. ${ }^{67}$ The ACGME program requirements for Family Medicine list none of these competencies ${ }^{8,9}$. If a FM program choose to follow the RCGFMR guideline recommendations within the framework of the ACGME requirements, their residents would have a significantly different ophthalmologic training experience than a those in a program not adhering to the RCGFMR recommendations.

It is the goal of the study to report GME family and internal medicine providers screening and examination practices, knowledge base, and referral patterns related to ophthalmologic concerns. Based upon the results, suggestions for potential improvements to resident training and clinical practices will be discussed as related to access to and quality of care. The proposed suggestions will also account for the potential to improve continuity of care and patient safety.

\section{Methods}

The data utilized for this study was gathered as part of a quality improvement project and was determined Institutional Review Board exempt according to Title 45 of the Code of Federal Regulations (45 CFR 46.101(b)(4)).

\section{Participants}

The study was conducted in 2018 by anonymously surveying family and internal medicine residents and attendings from multiple ambulatory clinic sites within a single GME system as part of a quality improvement project. The survey was designed to determine the incidence and methods of routine visual screening and what patient symptoms, medical histories, or complaints prompted referral to an ophthalmologist. The provider's knowledge base of common ocular diseases and systemic treatments with potential ocular complications was also assessed. The referral patterns of the FM/IM providers for ophthalmologic care was evaluated for rationale and location.

\section{Survey Development and Outcomes}

The survey consisted of 12 questions that either offered a choice of "yes" or "no" or prompted the provider to supply an answer(s) relating to either new patient or annual patient visits. The survey items that consisted of providers' write-in answers were grouped into broader categories for analysis. For example, providers who indicated "yes" to the question of "At the patient's annual exam, do you assess their visual status" were then asked to provide all methods they utilized to accomplish the assessment. Responses of pupil testing, fundoscopy, ocular motility testing where grouped under the category of "examination," whereas responses such as asking about visual changes, if the patient has seen an eye doctor this year, or if the patient noticed decreased vision were group under the category of "history." Responses that had a high incidence were reported as singular items rather than grouped into the corresponding category. Examples of such are visual field testing and corrective lens history in Table 2.

\section{Analysis}

Data was securely transmitted from Survey Monkey to an Excel spread sheet (Microsoft, Redmond, WA) stored on a secure server. Survey results were analyzed for descriptive statics. All results are reported as percentages. The percentages for survey items that allowed multiple responses are presented as aggregates. Due to the anonymous nature of the survey, comparisons between providers could not be performed, thus no $p$ values are reported. 


\section{Results}

The survey response rate was $45 \%$. The survey sample population consisted of 152 primary care physicians with the following distribution: 58 family practice residents; 10 family practice attendings; 76 internal medicine residents; 8 internal medicine attendings. Due to the anonymity of the survey, a more detailed demographic distribution of respondents could not be determined.

Nearly half the respondents (40\%) indicated they did not assess the visual status of their patients, nor did another member of the care team (63\%). A low percentage of providers (16\%) indicated ocular diseases was part of their past medical history evaluation. Fifty-two percent of respondents indicated they did query patients regarding visually related activities of daily living such as driving and watching television (Table 1).

The methods providers utilized to accomplish visual screening are detailed in Table 2. The screening could be performed by either the provider or a member of the care team, such as a medical assistant. The percentages shown in the table are aggregates as multiple responses were possible.

When patients reported visual complaints or visual deficiency was noted on exam, all providers (100\%) indicated they refer patients for ophthalmology evaluation. The majority of the providers either placed an order for referral or their office arranged the referral (88\%). Of those that not place an order for or have their office arrange a referral, only $42 \%$ recommended to the patient to arrange their own ophthalmology appointment (Table 3).

The primary referral location for ophthalmologic issues was the ophthalmology resident clinic within the GME system (43\%). There was a significant percentage of providers that indicated they did not know where to refer patients for ophthalmology evaluation (42\%). Referrals to ophthalmologists outside the GME system was infrequent (8\%) (Table 4).

Tables 5 contains the aggregate responses of primary care providers relating to what systemic conditions or medication usage would prompt them to seek an ophthalmology referral for a patient. All respondents (100\%) indicated they would refer diabetic patients for ophthalmology referral with hypertension (16\%) and rheumatic conditions (16\%) also prompting the highest rates of referral. Nearly half the respondents (42\%) were uncertain as to which medications should prompt a referral to ophthalmology.

\section{Discussion}

This study presents the vision screening, assessment, common ophthalmologic knowledge, and referral practices of primary care providers in a single GME ambulatory network and the potential impacts on the accesses to, quality, and continuity of care provided to patients. The continuously changing nature of residency programs and health systems structure offers a unique environment to study these vital measures. Regardless of the historical indicators used, or the level of training possessed, the results indicate the potential for improvement in vision screening, assessment, base knowledge, and referral for appropriate ophthalmologic care among GME primary care providers.

The results of this study share similarities to the Continuity of Care in Resident Outpatient Clinics: A Scoping Review of the Literature ${ }^{8}$. Examining continuity of care in resident clinics presents a challenge due to the rapid turnover in physicians and different levels of knowledge. The goal of the literature review was an attempt to understand the viable methods to nurture the continuity between resident physicians and patients. The results of this study can be used as a basis to cultivate the continuity of patient care between primary care providers and ophthalmologists within the GME network. This in turn could have a significant impact on the access to and quality of care provided to patients. 
It was encouraging that approximately $75 \%$ of patients received some form of visual screening at their annual examination (Table 1), even though none of the previously sited FM/IM specialty college guidelines mandate it be done. Visual screening could encompass anything from Snellen visual acuity testing to asking the patient if they have seen an eye doctor in the past year. However, a minority of respondents $(16 \%)$ indicated they asked patients about their family history of ocular disease. Family history of ocular disease is regarded as one of the primary risk factors in determining if an individual should have an ophthalmologic screening exam as indicated by the AAO. ${ }^{1}$ Similarly, visual acuity testing was only performed in one out of four patients (Table 2). A potential reason for the results are the different guidance criteria regarding vision screening and ocular disease assessment between specialty collages. The AAO recommends all individuals over age 40 have a complete ocular examination and those over age 65 a complete exam every one to two years. ${ }^{1}$ Further, individuals with risk factors that have potential visual impact should have a baseline examination. The AAFP and ACP do not include visual screening as part of an adult yearly examination for any age ${ }^{3-5}$. They also do not provide specific guidance for ophthalmologic evaluation for diabetic and hypertensive patient

When patients did receive vision screening and/or assessment indicating decreased acuity, all providers (100\%) reported they requested an ophthalmology referral (Table 3). However, not all arranged the referral for the patient (12\%), and the majority of those providers (58\%) indicated they did not communicate to the patient that they should seek an ophthalmology evaluation (Table 3). This calls into question how those who did not directly arrange an ophthalmology referral or suggest it to the patient felt that they "referred" the patient to ophthalmology. These results indicate that when primary care providers have data concerning a patient's visual status, they make appropriate referrals. This fact alone is strong evidence that visual screening should be part of the routine initial examination protocol for all adults and annually for individuals older than 65 years or those with historic or systemic risk factors.

In this particular survey population, it was interesting that a significant percentage of respondents indicated they did not know where to refer (42\%) their patients for ophthalmologic evaluation (Table 4). All FM residents within the surveyed GME system spend two weeks of their training rotating through the ophthalmology service in their PGY3 year. It is possible a large percentage of the respondents that indicated they did not know where to refer patients were junior residents that had not yet completed their ophthalmology rotation. Additionally, IM residents within the system do not rotate through the ophthalmology service. It may be of benefit that all primary care residents rotate with the ophthalmology service early in their training or are provided didactic instruction regarding ophthalmology referral criteria and locations within the GME system. This would increase access to care for patients with ophthalmologic concerns.

It is also notable that $7 \%$ of patients were referred to other medicine providers for ophthalmologic evaluation. Due to the anonymous nature of the survey, it is unknown if these referrals were from FM providers to IM providers or if a junior resident was referring the patient to a more senior resident. It is also possible this is a result of providers being unaware of ophthalmology referral services available within the GME system.

It was very encouraging that $100 \%$ of the respondents referred diabetic patients for ophthalmologic evaluation (Table 5). While the diabetic referral percentage is very encouraging, the percentage for hypertension was low (16\%). The AAO visual screening recommendations for individuals with hypertension are the same as those with $\mathrm{DM}^{1}$. According to the guidelines, the risk factors that should precipitate referral for a complete ophthalmic examination are DM, HTN, and family history of eye disease. It is interesting to note that some primary care providers considered cataracts and glaucoma "systemic" diseases. This suggests a potential knowledge gap within this area. As outlined in the "Knowledge" section of the RCGFMR, residents should understand ocular manifestations and complications of systemic diseases, as well as guidelines for appropriate intervals for vision evaluation. ${ }^{10}$ The inclusion of the later is surprising as the ACGME program requirements and college for FM physicians provides no guidance as to these intervals in healthy or diseased individuals. ${ }^{3,4}$ 
The "Knowledge" section of the RCGFMR also states that residents should understand the effects of drugs and toxins on ocular function and disease. ${ }^{6}$ Almost half the respondents (42\%) were unsure what medications could have potential ocular implications and warrant an ophthalmology referral for evaluation (Table 5). A low percentage (20\%) recognized that plaquenil requires ocular monitoring, specifically automatized visual field testing prior to starting medication and then annually while on the medication ${ }^{1}$. As detailed above, this represent a potential knowledge gap that could affect quality of care, as well as patient safety.

The anonymous design of the survey was a limiting factor in the study. The distributional data between family and internal medicine residents and attendings would provide another level of analysis. However, for this particular study, we felt the potential increase in response rate using the anonymous survey design would add more value to the results than being able to compare responses between provider types. In future studies, being able to obtain such acuity in the data would provide insight into if attendings were also unaware of referral guidelines and/or resources or if training level or residency type were the primary variables. As the design of ACGME resident training programs are top down, a deficiency in attending knowledge surrounding these topics could certainly influence the residents' knowledge base. Further, being able to compare the responses of residents that had an ophthalmology rotation versus those that did not within and between medicine specialties would also provide useful data on the effectiveness of specialty rotations in primary care GME training. This would have particular utility in determining how residents work in interspecialty teams to provide continuity of care, which can directly impact the quality of care and patient safety.

Based on the data, we propose two recommendations to improve primary care residents training in ophthalmic concerns and potentially increase access to and quality of care within a GME ambulatory health system. First, maximization of the historical indicators and visual screening used by primary care providers. Screening is a vital tool for physicians to gauge the health of our patients, thereby decreasing the likelihood of missing pathology. Every patient should be questioned about visual history and function at every annual or new patient visit. Visual acuity is recommended to be assessed at all initial visits or annually in those above 65 or with risk factors. Those who present with visual symptoms or complains should also receive Snellen acuity testing. Visual impairment is associated with falls in several studies, and those studies suggest patients having a screening at least every two years, and any refractive error corrected ${ }^{9,11}$. Due to the overall 1-year mortality being around $21.2 \%$ for hip fractures, annual assessment in susceptible populations would likely have an impact on quality of care and patient safety. ${ }^{12}$.

The second recommendation is education of primary care providers regarding ophthalmologic issues as outlined in the RCGFMR about the available ophthalmology referral options within the GME network at the onset of their training. To achieve this, annual didactics and interdisciplinary meetings between ophthalmology and primary care providers should be scheduled ${ }^{13}$. The interdisciplinary meetings are a two-fold solution. First, these meetings are an educational opportunity for specialists and primary care providers to share knowledge about topics. Second, to educate new providers within the GME network of available resources for referral and assistance in co-managing patients.

\section{Conclusion}

The data suggest that there are areas for improvement within GME ambulatory care networks regarding ophthalmologic screening, assessment, and referral. This analysis presents the rationale for modification of a GME primary care team's visual screening practices to improve care access and quality for patients requiring ophthalmologic services with a timely and appropriate referral. Similarly, there may be a benefit to provide further education to practitioners regarding specific indicators requiring ophthalmologic referral. In order to improve access, quality, and continuity of care within a GME ambulatory care network for ophthalmologic issues, additional education and training of primary care providers is necessary and should follow those outlined in the RCGFMR. Protocols for patient evaluations pertaining to vision screening, ocular history and evaluation, and referral should be implemented for all GME primary care physicians based 
upon age and risk factors. There maybe be added benefit of FM and IM residents having rotational experience with ophthalmology providers within the GME system for both medical knowledge and continuity of care.

\section{Declarations}

ETHICS: the authors of this paper have no ethical disclosures to report

FUNDING: The authors of this paper have no financial disclosures

CONFLICT OF INTEREST: The authors of this paper have no conflicts of interests related to the information in the article

DATA AVAILABILITY STATEMENT: The data of this research is available upon request by email

Competing Interests: To the best of our knowledge, the authors of this paper have no conflicts of interest or competing interests with the information enclosed in this paper.

Funding Info: no funding was provided for the subject of this paper.

Data Availability: data is available upon request

Author contribution: All authors have contributed to the writing of this paper.

Animal Research: No research was performed on animals.

Consent to Participate: All participates consented to fill out an anonymous survey by completing the survey.

Consent to Publish: the authors of this paper give the publisher the permission of the authors to publish the work after acceptance to the journal.

\section{References}

1. Turbert D. Eye Exam and Vision Testing Basics. Accessed April, 5th 2019, 2019. https://www.aao.org/eyehealth/tips-prevention/eye-exams-101

2. Force USPST. USPSTF A and B Recommendations. https://www.uspreventiveservicestaskforce.org/Page/Name/uspstf-a-and-b-recommendations/

3. Heidelbaugh JJ. The Adult Well-Male Examination. Am Fam Physician. Dec 15 2018;98(12):729-737.

4. Riley M, Dobson M, Jones E, Kirst N. Health maintenance in women. Am Fam Physician. Jan 1 2013;87(1):30-7.

5. Physicians ACo. Clinical Guidelines \& Recommendations. Accessed 03/10, 2020. https://www.acponline.org/clinical-information/guidelines

6. Physicians AAoF. Conditions of the Eye. Accessed 3/10, 2020. https://www.aafp.org/dam/AAFP/documents/medical_education_residency/program_directors/Reprint263_Eye.pdf

7. ACGME. ACGME Program Requirements for Graduate Medical Education in Family Medicine. Accessed 3/10, 2020. https://www.acgme.org/Portals/0/PFAssets/ProgramRequirements/120_FamilyMedicine_2019_TCC.pdf?ver=201906-13-074503-640

8. Walker J, Payne B, Clemans-Taylor BL, Snyder ED. Continuity of Care in Resident Outpatient Clinics: A Scoping Review of the Literature. J Grad Med Educ. Feb 2018;10(1):16-25. doi:10.4300/JGME-D-17-00256.1

9. Ivers RQ, Norton R, Cumming RG, Butler M, Campbell AJ. Visual impairment and risk of hip fracture. Am J Epidemiol. Oct 2000;152(7):633-9. 
10. Recommended Curriculum Guidelines for Family Medicine Residents: Conditions of the Eye. American Academy of Family Physicians. Accessed April, 5th, 2019.

https://www.aafp.org/dam/AAFP/documents/medical_education_residency/program_directors/Reprint263_Eye.pdf

11. Ivers RQ, Cumming RG, Mitchell P, Simpson JM, Peduto AJ. Visual risk factors for hip fracture in older people. J Am Geriatr Soc. Mar 2003;51(3):356-63.

12. Schnell S, Friedman SM, Mendelson DA, Bingham KW, Kates SL. The 1-year mortality of patients treated in a hip fracture program for elders. Geriatr Orthop Surg Rehabil. Sep 2010;1(1):6-14. doi:10.1177/2151458510378105

13. Gwynne M, Page C, Reid A, Donahue K, Newton W. What's the Right Referral Rate? Specialty Referral Patterns and Curricula Across I3 Collaborative Primary Care Residencies. Fam Med. Feb 2017;49(2):91-96.

\section{Tables}

Table 1: Frequency of vision screening and ocular disease assessment at primary care visit.

\begin{tabular}{|c|c|c|}
\hline Question & Yes & No \\
\hline Do you assess visual status? & $60 \%$ & $40 \%$ \\
\hline If you do not assess visual status, does a member of your team assess? & $37 \%$ & $63 \%$ \\
\hline Does your patient history include ocular disease or blindness? & $16 \%$ & $84 \%$ \\
\hline $\begin{array}{l}\text { Do you inquire about daily activities such as reading, identifying medicine labels, close work, or } \\
\text { watching television? }\end{array}$ & $52 \%$ & $48 \%$ \\
\hline
\end{tabular}

Table 2: Methods of visual screening utilized by primary care practices. Percentages are aggregates, indicating each modality the respondent provided.

\begin{tabular}{|ll|}
\hline Visual acuity testing & $24 \%$ \\
\hline Examination & $29 \%$ \\
\hline Visual Field Testing & $6 \%$ \\
\hline History & $35 \%$ \\
\hline Corrective Lens History & $6 \%$ \\
\hline
\end{tabular}

Table 3. Referral patterns of primary care providers.

\begin{tabular}{|c|c|c|}
\hline Question & Yes & No \\
\hline $\begin{array}{l}\text { If a patient reports changes in their vision or evaluation reveals a change, do you refer them for } \\
\text { ophthalmology evaluation? }\end{array}$ & $100 \%$ & $0 \%$ \\
\hline Do you place a medical order/arrange for a referral? & $88 \%$ & $12 \%$ \\
\hline $\begin{array}{l}\text { If the referral is not arranged by your office, do you make a recommendation for a patient to arrange } \\
\text { their own? }\end{array}$ & $42 \%$ & $58 \%$ \\
\hline
\end{tabular}

Table 4. Ophthalmology referral locations of primary care providers. 


\begin{tabular}{|ll|}
\hline Internal Medicine clinic & $7 \%$ \\
\hline Private Ophthalmologist & $8 \%$ \\
\hline Ophthalmology Resident clinic & $43 \%$ \\
\hline Does not know where to refer & $42 \%$ \\
\hline
\end{tabular}

Table 5. Systemic conditions and medications that would prompt a referral to ophthalmology. Percentages are aggregates indicating the respondents who named a specific condition.

Conditions

\begin{tabular}{|ll|}
\hline Fungemia & $4 \%$ \\
\hline Pituitary adenoma & $4 \%$ \\
\hline Multiple Sclerosis & $4 \%$ \\
\hline Herpes Involving the face or nose & $4 \%$ \\
\hline Hemochromatosis & $4 \%$ \\
\hline Connective tissue disease & $4 \%$ \\
\hline Cataracts & $8 \%$ \\
\hline Hypertension & $16 \%$ \\
\hline Rheumatic Disease & $16 \%$ \\
\hline Glaucoma & $20 \%$ \\
\hline Diabetes Mellitus & $100 \%$ \\
\hline
\end{tabular}

Medications

\begin{tabular}{|ll|}
\hline Steroids & $5 \%$ \\
\hline Quetiapine (Seroquel $\AA$ ) & $5 \%$ \\
\hline Amiodarone & $10 \%$ \\
\hline Glaucoma Medications & $20 \%$ \\
\hline Rheumatologic Medications & $23 \%$ \\
\hline Did Not Know & $42 \%$ \\
\hline
\end{tabular}

